\title{
Inhibiting miRNA in Caenorhabditis elegans using a potent and selective antisense reagent
}

\author{
Genhua Zheng1, Victor Ambros² and Wen-hong Li*1
}

\begin{abstract}
Background: Antisense reagents can serve as efficient and versatile tools for studying gene function by inhibiting nucleic acids in vivo. Antisense reagents have particular utility for the experimental manipulation of the activity of microRNAs (miRNAs), which are involved in the regulation of diverse developmental and physiological pathways in animals. Even in traditional genetic systems, such as the nematode Caenorhabditis elegans, antisense reagents can provide experimental strategies complementary to mutational approaches. Presently no antisense reagents are available for inhibiting miRNAs in the nematode C. elegans.
\end{abstract}

Results: We have developed a new class of fluorescently labelled antisense reagents to inhibit miRNAs in developing worms. These reagents were synthesized by conjugating dextran with 2'-O-methyl oligoribonucleotide. The dextranconjugated antisense reagents can be conveniently introduced into the germline of adult hermaphrodites and are transmitted to their progeny, where they efficiently and specifically inhibit a targeted miRNA in different tissues, including the hypodermis, the vulva and the nervous system. We show that these reagents can be used combinatorially to inhibit more than one miRNA in the same animal.

Conclusion: This class of antisense reagents represents a new addition to the toolkit for studying miRNA in C. elegans. Combined with numerous mutants or reporter stains available, these reagents should provide a convenient approach to examine genetic interactions that involve miRNA, and may facilitate studying functions of miRNAs, especially ones whose deletion strains are difficult to generate.

See related research article: $\underline{h t t p}: / /$ jbiol.com/content/9/3/20

\section{Background}

MicroRNAs (miRNAs) are single strand RNA molecules 21-23 nucleotides long that play important roles in many biological processes through regulating gene expression [1]. In animal cells, miRNAs act primarily by inhibiting mRNA translation and/or stability through a process involving partial complementary base-pairing with sequences at the $3^{\prime}$-untranslated region (3' UTR). Numerous miRNAs have been identified. To study their functions, antisense reagents against miRNAs have been developed as a reverse genetics tool. Synthetic oligonucleotide analogues, including 2'-O-methyl oligoribonucleotides [2], locked nucleic acids [3], 2'-O-methoxyethyl oligoribonucleotides [4], and morpholinos [5], have been

\footnotetext{
*Correspondence: wen-hong.li@UTSouthwestern.edu

1 Departments of Cell Biology and of Biochemistry, University of Texas

Southwestern Medical Center, 5323 Harry Hines Blvd, Dallas, TX 75390-9039, USA

Full list of author information is available at the end of the article
}

tested. These antisense nucleotide analogues have been used to knock down miRNAs in cultured cells [2-4] and in live animals including zebrafish [5], D. melanogaster [6] and mice [7].

Caenorhabditis elegans has long been used as a model organism for studying the regulation and function of small non-coding RNA molecules, and yet no antisense reagents are available to reliably inhibit miRNAs in worms. Such a technique would be very useful for studying functions of miRNAs whose deletion strains are difficult to generate; for example, mutations causing lethality or sterility [8]. In addition, to dissect functions of individual miRNAs that are clustered together, or to block intronic miRNAs $[8,9]$ without perturbing the function of the corresponding protein-coding genes, antisense reagents would offer a convenient approach to circumvent the limitation of using deletion strains. 


\section{Results and discussion}

Previous studies by Hutvagner et al. showed that antisense 2'-O-methyl oligoribonucleotide injected into $C$. elegans larvae could inhibit functions of a miRNA [2]. However, injection of worm larvae is technically very demanding, and so the larval injection of anti-microRNA oligonucleotides has not been employed for C. elegans except the original report [2]. A much more straightforward approach would be to inject the antisense compound into the syncitial gonad of hermaphrodites using the standard injection methods employed for the DNA transformation of $C$. elegans [10] so that the reagent would inhibit a targeted microRNA during the embryonic and larval development of the injected hermaphrodite's progeny. A previous attempt at this approach using 2'-O-methyl oligoribonucleotides failed to produce the expected phenotype [2]. We suspect that the cellular uptake, retention or distribution properties of unconjugated 2'-O-methyl oligoribonucleotides might not be optimal for an efficient inhibition of miRNA in worms. To develop a robust antisense technique to inhibit miRNAs in C. elegans, we explored modifying 2'-O-methyl oligoribonucleotides through conjugation with dextran, a polysaccharide that is nontoxic, inert and soluble in aqueous solutions. Dextrans are well retained in cells over a long period of time and dextran-dye conjugates have been widely used for cell labelling and cell lineage tracing [11]. To conjugate dextran with 2'-O-methyl oligoribonucleotides, we first reacted dextran amines (molecular weight $40 \mathrm{KDa}, \sim 8$ amines/dextran) with a water-soluble bifunctional linker, MAL-dPEG4-NHS ester in order to produce dextran- $\left[(\mathrm{PEG})_{4}-\mathrm{MAL}\right]_{8}$ (Figure 1a). This thiolreactive intermediate was then conjugated with eight equivalents of antisense 2'-O-methyl oligoribonucleotides containing a 5 -thiol group. The resulting product, dextran-(as-2'OMe $l i n-4)_{8}$, or D-(as-2'OMe $\left.l i n-4\right)_{8}$, contains, on average, eight copies of 2'-O-methyl oligoribonucleotides complementary to lin-4, the founding miRNA first identified in C. elegans [12,13].

To apply dextran-(as-2'OMe $l i n-4)_{8}$ to inhibit lin-4 in vivo, we injected the compound into gonads of adult hermaphrodites. Dextran-rhodamine $(40 \mathrm{KDa})$ was coinjected as a fluorescent marker. About $16 \mathrm{~h}$ after injection, we collected rhodamine-labeled embryos $(n=50)$ under a fluorescence dissection scope. When these embryos reached adulthood, we scored for the egg laying defective (Egl) phenotype. In C. elegans, lin-4 is required during larval development to control the timing and pattern of cell division in the hypodermis of larva stage 1 (L1) and stage 2 (L2). lin-4 loss of function mutants $(l i n-4(l f))$ display inappropriate reiterations of early fates at late developmental stages and show a retarded heterochronic phenotype in adults in the form of the absence of adult structures (such as vulva) and the failure of egg-laying $[12,13]$.

When using an injection with a concentration of $50 \mu \mathrm{M}$ (all concentrations refer to the total concentration of 2'O-methyl oligoribonucleotides in the sample as determined from the ultraviolet (UV) absorption at $260 \mathrm{~nm}$ ) dextran-(as-2'OMe $l i n-4)_{8}$ was effective in inhibiting lin-4 and caused Egl in about $70 \%$ of worms (Figure 1b-d). Raising the injection concentration to $100 \mu \mathrm{M}$ or above increased Egl to over 90\% in the labelled worms. In contrast, antisense 2'-O-methyl oligoribonucleotides that is not conjugated to dextran only had a small effect, even at $200 \mu \mathrm{M}$ (Figure 1b). In order to examine the specificity of dextran-(as-2'OMe $l i n-4)_{8}$ in inhibiting lin-4, we prepared two control dextran conjugates, dextran-(as-2'OMe $m i R$ $237)_{8}$ and dextran-(s-2'OMelin-4) $)_{8}$. Dextran-(as-2'OMe $m i R-$ 237) ${ }_{8}$ contains 2'-O-methyl oligoribonucleotides complementary to $m i R-237$, a miRNA of the lin-4 family with similar, but not identical, sequence as lin-4. Dextran-(s${ }^{2} \mathrm{OMe} /$ in -4$)_{8}$ contains lin-4 sequence (sense). We did not observe Egl phenotypes, or other abnormalities, in worms labelled with either of these two control oligonucleotides (Figure 1b) which confirmed that dextran-(as$\left.2^{\prime} \mathrm{OMe} l i n-4\right)_{8}$ inhibits $l i n-4$ in a sequence specific manner, also suggesting that worms tolerate dextran conjugates of 2'-O-methyl oligoribonucleotides fairly well.

In dextran-(as-2'OMelin-4), each dextran molecule is conjugated to eight copies of antisense 2'-O-methyl oligoribonucleotides. Having a high density of oligonucleotides on the surface of dextran molecule might increase the steric hindrance and compromise the hybridization efficiency of the antisense oligonucleotide to its target miRNA. In order to test whether we could improve the potency of these dextran conjugated antisense reagents by varying the coupling stoichiometry, we decreased the amount of 2'-O-methyl oligoribonucleotides used for conjugation. In addition, we also linked a fluorescent label (rhodamine B isothiocyanate) with dextran so that we could visualize the distribution of these antisense reagents directly. We prepared two rhodamine-dextran (Rhdextran) conjugates of 2'-O-methyl oligoribonucleotides, Rhdextran-(as-2'OMe $l i n-4)_{4}$ and Rhdextran-(as2 'OMe $l i n-4)_{1}$, by varying the equivalents of 2'-O-methyl oligoribonucleotides added to the conjugation reaction (Figure 2). Each Rhdextran-(as-2'OMelin-4) $)_{4}$ or Rhdextran-(as$\left.2^{\prime} \mathrm{OMe} / \mathrm{in}-4\right)_{1}$ on average contains four or one copy of lin-4 antisense 2'-O-methyl oligoribonucleotide, respectively (Figures 2 and 3a). These two dextran conjugates were comparably efficient in inhibiting lin-4, yet both were much more potent than dextran-(as-2'OMe $l i n-4)_{8}$. At $20 \mu \mathrm{M}$ or above, both ${ }^{\mathrm{Rh}}$ dextran-(as-2'OMe $\left.l i n-4\right)_{4}$ and Rhdextran-(as- 

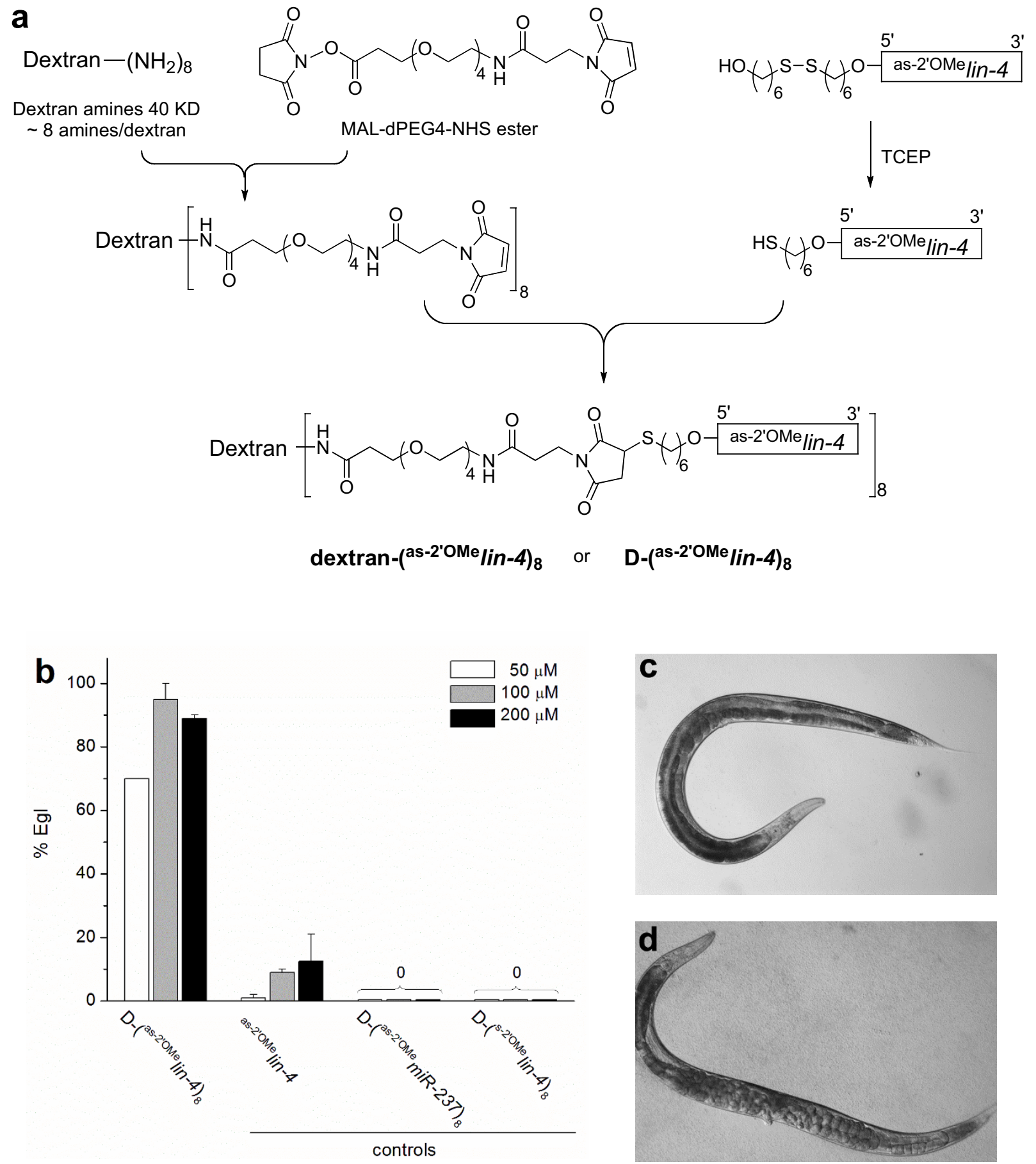

Figure 1 Inhibit miRNA in Caenorhabditis elegans using a dextran-conjugate of antisense 2'-O-methyl oligoribonucleotide. (a) Chemical synthesis of dextran-(as-2'OMe/in-4) ${ }_{8}$, or D-(as-2'OMe/in-4) ${ }_{8}$, a dextran conjugate of an antisense reagent against lin-4. (b) Dose response of D-(as-2'OMe/in-4) ${ }_{8}$ in causing egg laying defectives (Egl) via lin-4 inhibition. Controls include as-2'OMe/in-4, a lin-4 antisense 2'-O-methyl oligoribonucleotide without dextran; D-(s-2'OMe $/$ in-4) ${ }_{8}$ and D-(as-2'OMemiR-237) ${ }_{8}$, dextran conjugates containing either lin-4 sense or miR-237 antisense 2'-O-methyl oligoribonucleotide. Error bars are standard errors of two independent injections. Each time eight worms were injected and 50 or more labelled embryos from injected worms were scored for Egl when they reached adults. (c, d) Example images of a normal worm (c) and an Egl worm (d) labelled with dextran-(as-2'OMelin-4) 

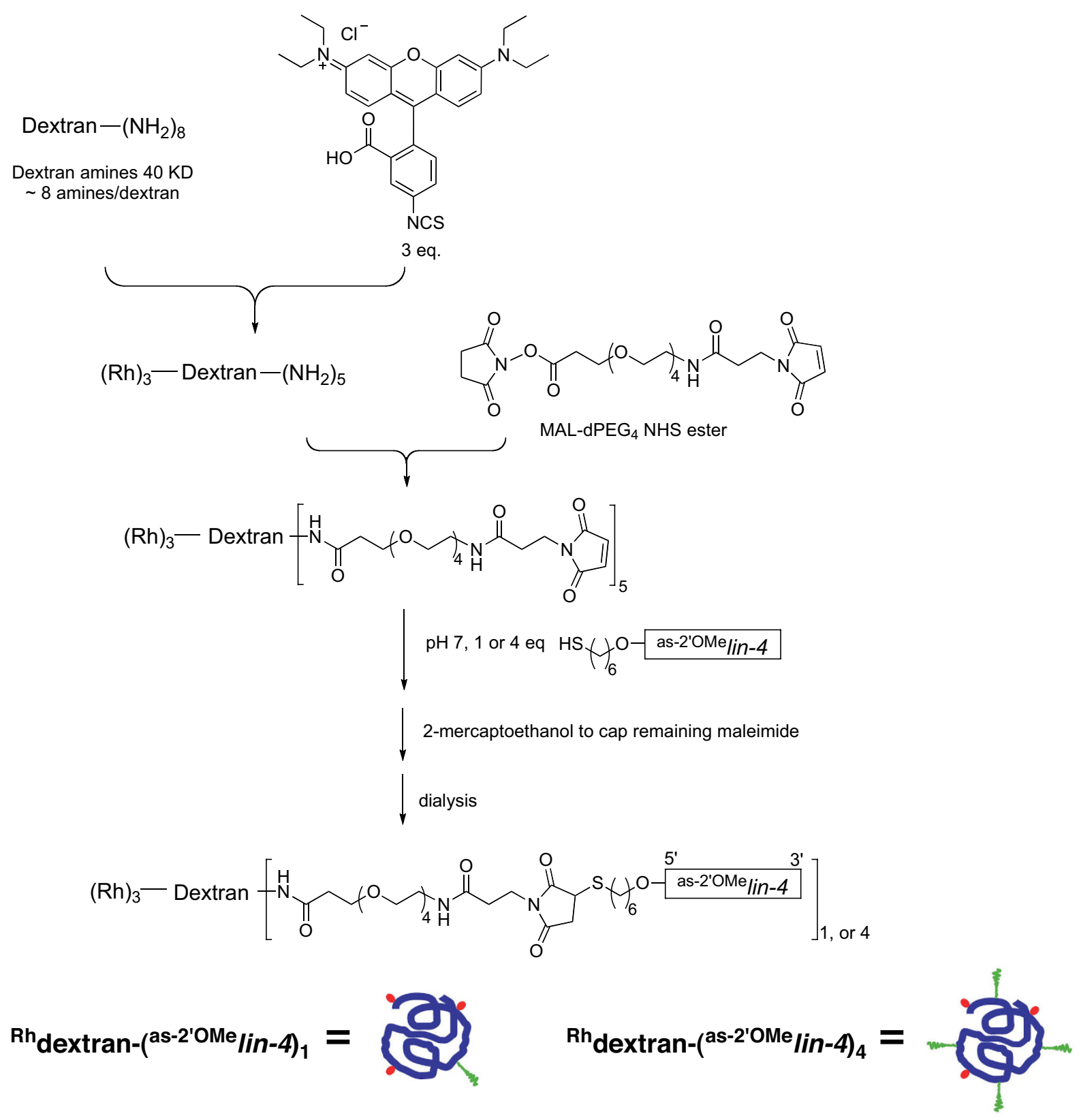

Figure 2 Synthesis of rhodamine labelled dextran conjugates of 2'-O-methyl oligoribonucleotide. During conjugation, different equivalents

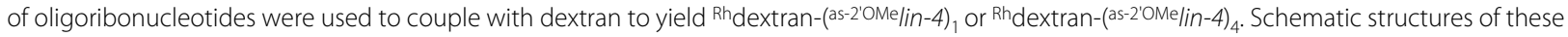
products are shown at the bottom, with the heavy blue line, wavy green line and the red dot representing dextran, 2'-O-methyl oligoribonucleotide and rhodamine, respectively. $\mathrm{Rh}=$ rhodamine; ${ }^{\mathrm{Rh}}$ Dextran $=$ rhodamine labeled dextran.

2'OMe lin-4) ${ }_{1}$ caused Egl in nearly $100 \%$ of labelled worms (Figure 3b). In contrast, dextran-(as-2'OMe $l i n-4)_{8}$ was completely ineffective at $20 \mu \mathrm{M}$. In addition, fluorescence imaging of Rhdextran-(as-2'OMelin-4) ${ }_{1}$ confirmed that the conjugate was localized fairly evenly in the cytosol after being taken up by cells.
In order to confirm that these antisense reagents act specifically by inhibiting lin-4, we examined several molecular and cellular markers to characterize the development of animals labelled with Rhdextran-(as-2'OMelin$4)_{1}$ : (1) the formation of vulval structure; (2) adult specific alae formation and col-19 expression; and (3) the stagespecific seam cell division programmes. 
a

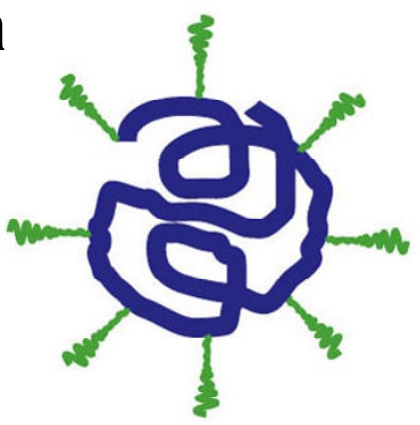

dextran-( $\left.{ }^{\text {as-2'OMe }} / \mathrm{in}-4\right)_{8}$

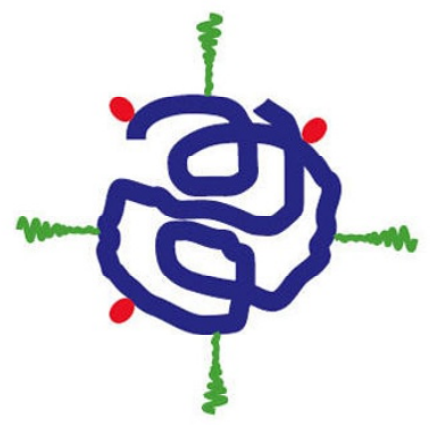

${ }^{R h}$ dextran-(as-2'OMe $\left./ i n-4\right)_{4}$

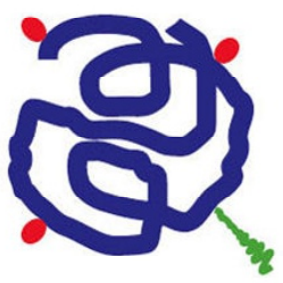

${ }^{R h}$ dextran-( ${ }^{\text {as-2'OMe }} /$ in-4 $)_{1}$

b
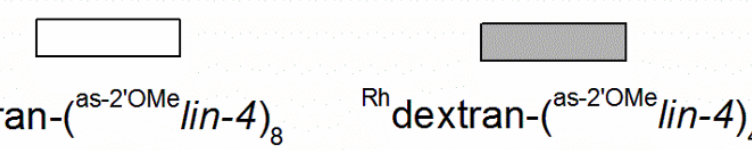

${ }^{\mathrm{Rh}}$ dextran-( ${ }^{\text {as-2'OMe }}$ lin-4)

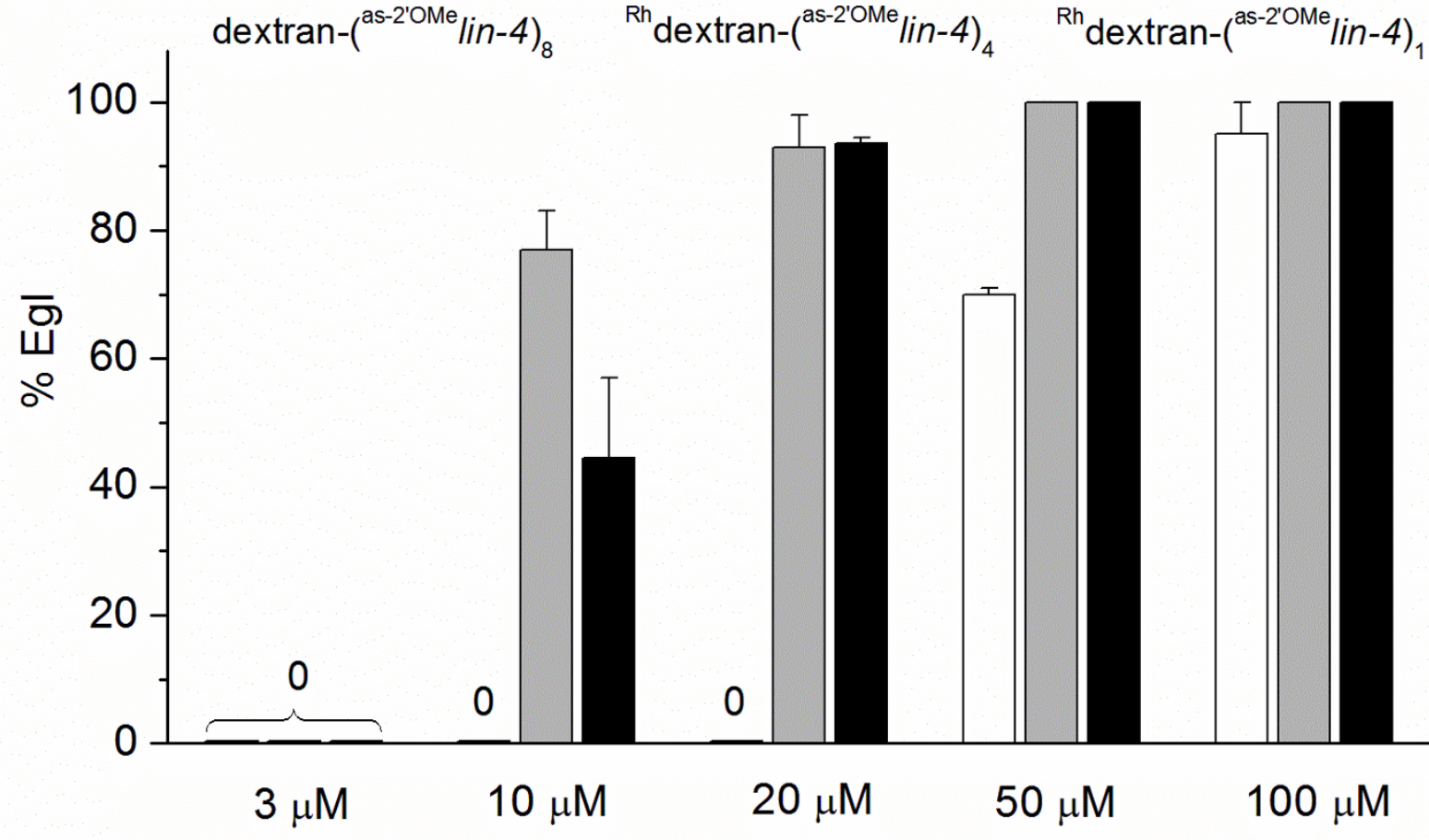

Figure 3 Coupling stoichiometry of 2'-O-methyl oligoribonucleotide affects the potency of dextran-conjugated antisense reagents. (a) Schematic structures of dextran-(as-2'OMe/in-4) ${ }_{8}{ }^{\text {Rh }}$ dextran-(as-2'OMe/in-4) ${ }_{4}$ and Rhdextran-(as-2'OMe/in-4). The heavy blue line, wavy green line and the red dot symbolize dextran, 2'-O-methyl oligoribonucleotide and rhodamine, respectively. (b) Dose response of dextran-(as-2'OMe/in-4) ${ }_{8}{ }^{\text {Rh }} \mathrm{dextran}^{\mathrm{C}}$ (as-2'OMelin-4) ${ }_{4}$, and Rh dextran-(as-2'OMe/in-4) in causing egg laying defectives. Error bars are standard errors of two independent experiments. Each time we scored at least 50 worms labelled with an antisense reagent.

The egg-laying defect of lin-4(lf) was due to inappropriate vulva development. In control animals, vulva morphogenesis is evident by early L4. By mid-L4 stage, the developing vulva displays a characteristic structure reminiscent of a Christmas tree (Figure 4a). In contrast, the vulva structure was missing in a lin-4(0) mutant, lin4(e912) (Figure 4b). Wild type animals labelled with Rhdextran-(as-2'OMelin-4) ${ }_{1}$ showed the similar vulvaless anatomy as lin-4(e912) (Figure 4c), consistent with lin-4 inhibition by ${ }^{\mathrm{Rh}}$ dextran-(as-2'OMe $/$ in-4) . $_{1}$. 


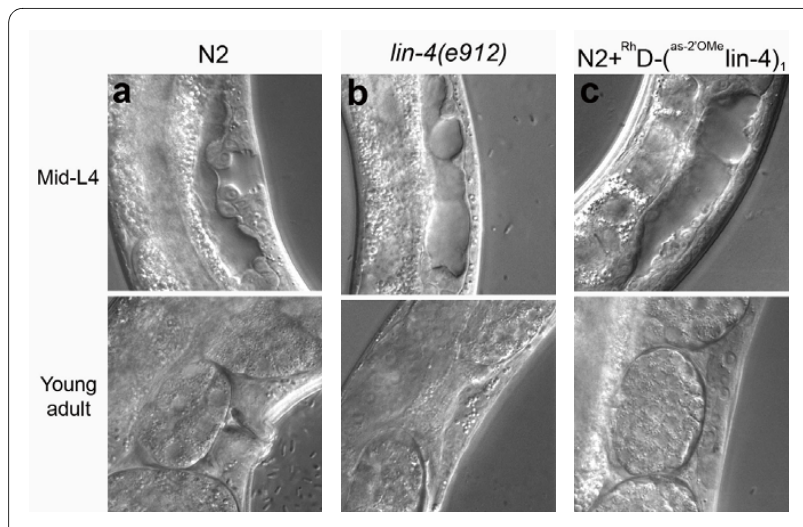

Figure 4 lin-4 inhibition with ${ }^{\mathrm{Rh}}$ dextran-(as-2'OMelin-4) ${ }_{1}$ disrupted vulva formation similarly as in a lin-4(0) mutant. The vulval structures in mid-L4 (top) and in adult (bottom) are well defined in control animals (a). Vulva failed to form in lin-4(e912) (b) and in lin-4 knockdown (c). The penetrance of vulval defect in (c) was $100 \%(n=45)$ with $50 \mu \mathrm{M}$ of Rh dextran-(as-2'OMe/in-4) .

Besides affecting the development of vulval cells, lin-4 also controls stage-specific lateral hypodermal cell fates. At the L4 molt, lateral seam cells exit cell cycle and form alae. At about the same time, hypodermal cells start to express an adult specific green fluorescence protein (GFP) reporter, col-19::GFP (Figure 5a, b) [14,15]. Animals labelled with Rhdextran-(as-2'OMe $l i n-4)_{1}(50 \mu \mathrm{M}$ injection concentration) failed to form alae at the L4 molt $(100 \%, n=20)$ and showed no col-19::GFP expression (100\%, $n=56$; Figure 5c, d). Interestingly, $10 \mathrm{~h}$ later, both

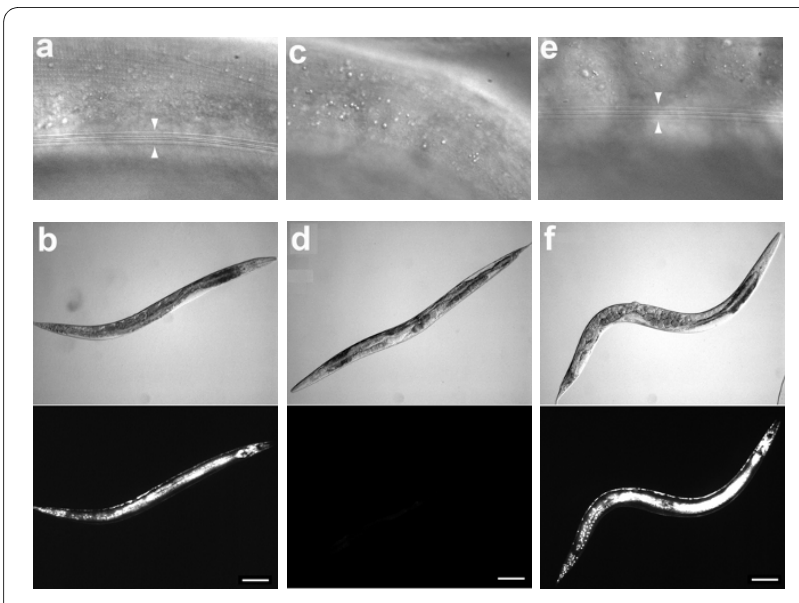

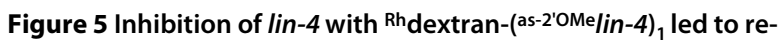
tarded development. $(\mathrm{a}, \mathrm{b})$ Control young adult animals $(\sim 55 \mathrm{~h}$ posthatching) showed normal alae [(a) highlighted by arrow heads] and col-19::GFP expression [(b) top - bright field; bottom - green fluorescence protein (GFP)]. (c-f) Young adult animals ( $55 \mathrm{~h}$ post-hatching) labelled with Rh dextran-(as-2'OMe/in-4) 1 (50 $\mu \mathrm{M}$ injection concentration) displayed no alae (c) or col-19::GFP (d). 10 hours later ( 65 h posthatching), both alae (e) and col-19::GFP (f) appeared in these animals. scale bar $=0.1 \mathrm{~mm}$.
col-19::GFP (100\%, $n=56)$ and alae $(100 \%, n=19)$ were observed in these animals (Figure $5 \mathrm{e}, \mathrm{f}$ ). Increasing the dose of Rhdextran-(as-2'OMelin-4) ${ }_{1}(150 \mu \mathrm{M}$ injection concentration) prolongs the duration of lin- 4 inhibition, such that at $10 \mathrm{~h}$ post-L 4 molt, only $1.8 \%$ of labelled worms showed col19::GFP $(n=55)$; about $60 \%$ of these animals displayed col19::GFP $15 \mathrm{~h}$ later (or $25 \mathrm{~h}$ post-L4 molt). These results suggest that lin-4 knockdown with ${ }^{R h} \mathrm{dex}-$ tran-(as-2'OMelin-4) 1 caused these animals to go through extra larval stages (L5 and L6) before they entered adult. This is consistent with a reduction in (but not complete elimination of) lin-4 function.

During larval development, the lateral hypodermal seam cells follow a characteristic division pattern. After each division, one of the daughter cells fuses with the hypodermis (hyp7) and the other daughter cells divides again at a later stage until it terminally differentiates at the L4 molt (Figure 6a) [16]. Seam cells of lin-4(e912) animals repeat the L1 fates in hypodermal cell lineages and are unable to exit the cell cycle at the L 4 molt. In order to determine how seam cell development is altered in lin-4 knockdown animals, we followed the seam cell division pattern by observing GFP-labelled seam cells (wIs51, strain JR667). Both L1 and L2 divisions in lin-4 knockdown appeared to be normal, with each $\mathrm{V}$ cell (including $\mathrm{V} 1$ - V4 and V6) divided once in L1 $(100 \%, n=10$, data not shown) and twice in L2 (100\%, $n=10$; Figure 6b, c). However, in early L3, V cells in lin-4 knockdown repeated the L2 division pattern, so that each $\mathrm{V}$ cell gave rise to twice as many daughter cells as the corresponding seam cells of the control animals (100\%, $n=6$; Figure 6d, e). This pattern of reiterated L2 seam cell division is consistent with a reduced, but not absent, activity of $l i n-4$, possibly resulting in an intermediate level of lin-14 over expression [17].

Finally, since $l i n-4$ functions through lin-14, mutations in lin-14 should suppress the phenotype of lin-4 knockdown. Indeed, at $20^{\circ} \mathrm{C}$, inhibition of lin-4 in lin-14(n179), a lin-14 nonnull mutant, with Rhdextran-(as-2'OMelin-4) (50 $\mu \mathrm{M}$ injection concentration) only caused Egl in $2.6 \%$ of labelled worms $(n=190)$ and all the examined young adult worms displayed normal alae $(n=30)$.

Together, these data showed that Rhdextran-(as-2'OMelin4) ${ }_{1}$ caused developmental retardation consistent with lin4 knockdown, confirming its efficacy and specificity in inhibiting lin-4 during development.

In order to test whether these conjugated antisense agents can be used to inhibit other miRNAs in worms, we prepared Rhdextran-(as-2'OMe $l s y-6)_{1}$ and Rhdextran-(as2 'OMelet-7) $)_{1}$ using the same procedure as for making Rhdextran-(as-2'OMelin-4) ${ }_{1}$. These two dextran conjugates were designed to block $l s y-6$ and let-7, respectively, two miRNAs of known functions in C. elegans. 

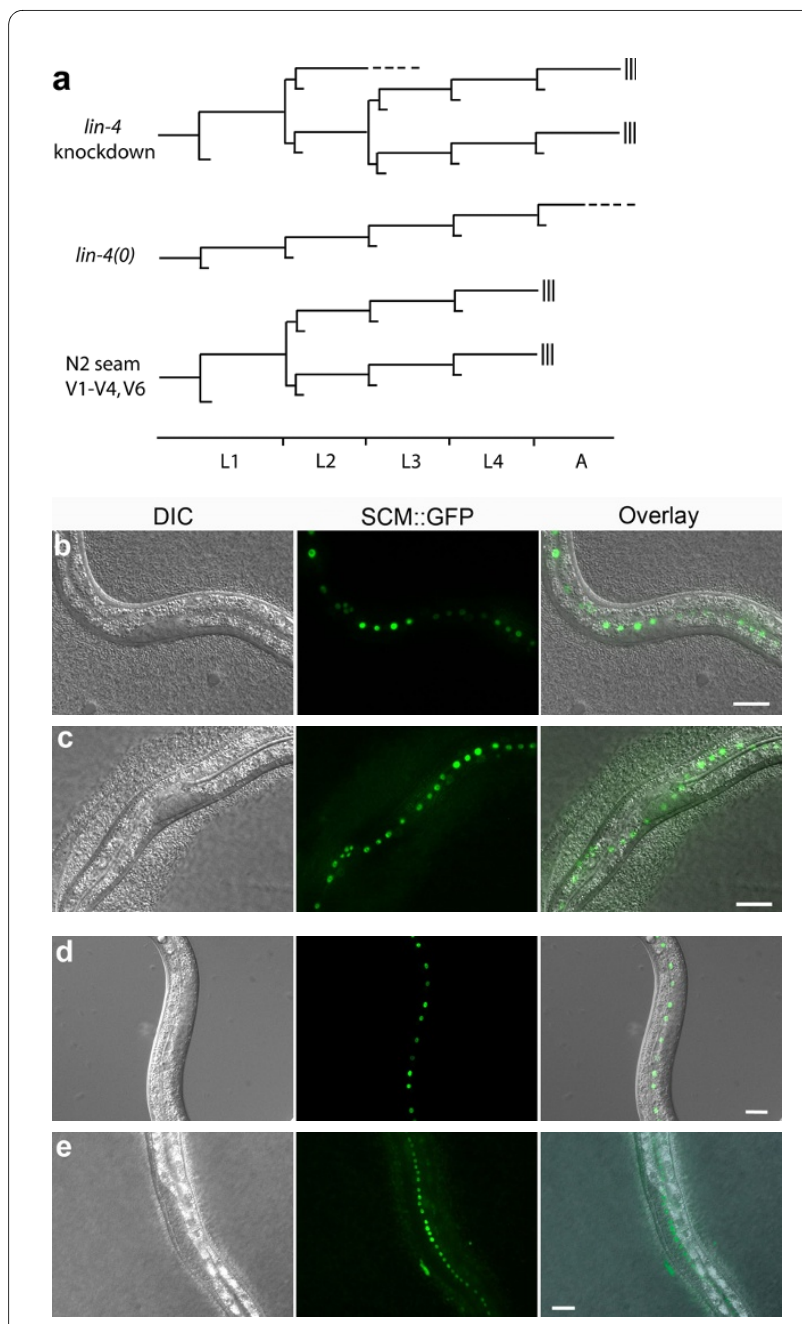

Figure 6 lin-4 knockdown caused repetition of L2 seam cell division pattern in L3. (a) Seam cell lineage for V1 - V4 and V6 of wild type, lin-4(0) and lin-4 knockdown by Rhdextran-(as-2'OMe/in-4), at $50 \mu \mathrm{M}$ injection concentration. $(b, c)$ In early $L 2$, each N2 seam cell gave rise to a quartet of daughter cells after two successive divisions in both N2 (b) and in lin-4 knockdown (c). (d, e) In early L3, each Vn cell of lin-4 knockdown repeated $\mathrm{L} 2$ programme, giving rise to twice as many daughter cells (e) as those of control animals (d). Each image is representative of at least six animals (see text). Scale bar $=20 \mu \mathrm{m}$.

The $l s y-6$ microRNA regulates left-right asymmetry of ASE neurons, a pair of chemosensory neurons that share many bilaterally symmetrical features, yet differ in their ability to discriminate different ions by expressing distinct sets of chemoreceptors of the gcy gene family [18]. lsy- 6 is only present in ASEL (left ASE) of adult worms. It promotes $g c y$-7 expression through repression of $\operatorname{cog}-1$, a transcription factor that negatively regulates $g c y-7$ expression [18]. In order to score the inhibition of $l s y-6$ activity, we used two reporter strains expressing GFP in either ASEL ( $g c y$-7prom::gfp, strain OH3191) or ASER (right ASE; $g c y$-5 prom:: $g f p$, strain OH3192). Rhdextran-(as- $\left.2^{\prime} \mathrm{OMe} l s y-6\right)_{1}$ was highly effective in inhibiting $l s y-6$. At the injection concentration of only $3 \mu \mathrm{M}$, the compound inhibited $l s y-6$ with nearly $100 \%$ penetrance, assayed by the repression of $g c y$-7prom:::gfp expression in ASEL (Figure 7a), and by the induction of ectopic $g c y$-5 prom:: $g f p$ expression in ASEL (Figure 7b).

Another miRNA, let-7, controls the larval-to-adult transition by repressing the translation of $l i n-41$ and $h b l$ 1. Mutations in let-7 lead to a retarded terminal differentiation of seam cells, which results in the elimination of alae and a bursting of the animal at the vulva as the animal undergoes the fourth molt [19]. We observed a dosedependent bursting or no-alae phenotype, consistent with inhibition of let-7 activity in the progeny of hermaphrodites injected with ${ }^{\mathrm{Rh}}$ dextran-(as-2'OMelet-7) ${ }_{1}$ (Figure 8). When ${ }^{\mathrm{Rh}}$ dextran-(as-2'OMelet-7) ${ }_{1}$ was injected at 20 $\mu \mathrm{M}$, it caused bursting vulva in about $40 \%$ of labelled worms and eliminated the formation of alae in nearly $80 \%$ of worms. Raising the concentration of Rhdextran-(as${ }_{2}$ 'OMelet-7) to $50 \mu \mathrm{M}$ or above increased the penetrance, and none of the labelled animals showed alae when they reached young adults. In contrast, an injection of Rhdextran-(as-2'OMe $m i r-84)_{1}$ directed against another let-7 family microRNA caused no observable phenotype. Since mir-84 mutations do not cause visible phenotypes [20], this result is consistent with these antisense reagents inhibiting the targeted miRNA with high specificity. Further, in a lin-41 nonnull mutant, lin-41(ma104), Rhdextran-(as-2'OMelet-7) $)_{1}(50 \mu \mathrm{M})$ failed to produce the bursting

a
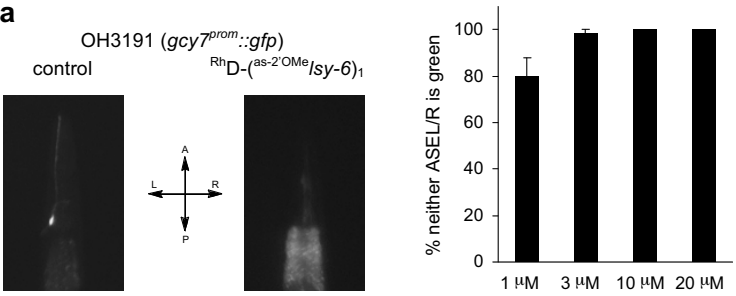

b
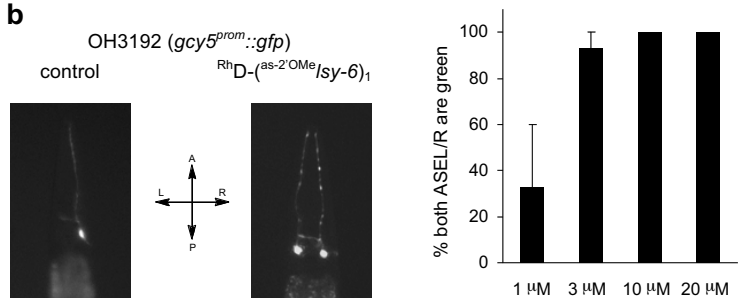

Figure 7 Inhibit Isy- 6 in neuronal cells with a dextran conjugated antisense reagent. (a) Dose response of Rh dextran-(as-2'OMe/sy-6) in repressing gcy-7prom::.gfp expression in ASEL(left ASE). (b) Dose response of Rh dextran-(as-2'OMe/sy-6) in causing ectotopic gcy-5 prom:.:gfp expression in ASEL. $L=$ left; $R=$ right; $A=$ anterior; $P=$ posterior 


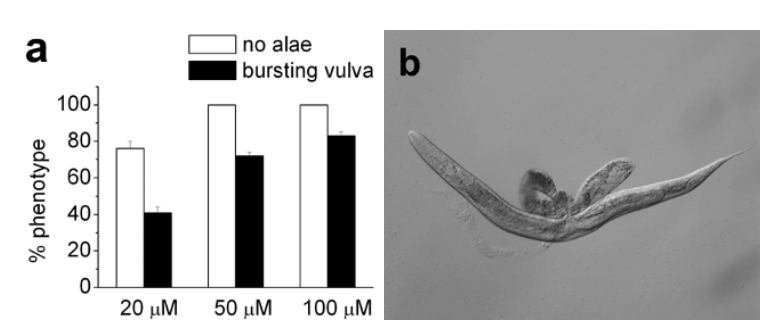

Figure 8 Inhibit let-7 with ${ }^{\mathrm{Rh}}$ dextran-(as-2'OMe/et-7) $)_{1}$ (a) Dose response of dextran-(as-2'OMelet-7), in causing bursting vulva or no alae formation. Error bars are standrad errors of two independent injections. After each injection, 50 or more embryos from injected worms were randomly picked and scored for bursting vulva or absense of alae when they reached adults. (b) An example image showing the bursting vulva phenotype.

vulva or alae defect in labelled worms $(n=12)$. This again suggested that these antisense reagents acted specifically by blocking their corresponding microRNAs.

In order to confirm that this class of antisense reagents is also effective in inhibiting microRNAs during embryo development, we prepared Rhdextran-(as-2'OMemir-42) against mir-42, a member of the mir-35 family which consists of eight microRNA genes of similar sequences. This family of microRNAs is expressed only during embryogenesis [21] and functions redundantly to control embryonic development $[8,22]$. Deletion of the seven microRNAs (mir35 - 41, strain MT14119) leads to a temperature-sensitive late embryonic or L1 lethal phenotype. At $15^{\circ} \mathrm{C}$, about $10 \%$ of the embryos displayed this phenotype, while the remaining $90 \%$ developed normally (Figure 9). In this mir35-41 null background, inhibition of

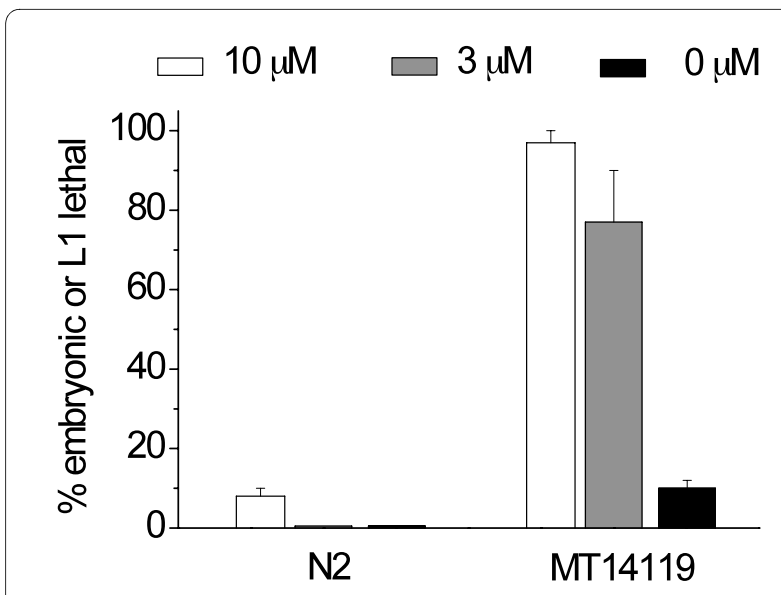

Figure 9 Inhibit mir-42 with ${ }^{\mathrm{Rh}}$ dextran-(as-2'OMe mir-42) ${ }_{1}$ during embryogenesis. Error bars are standard errors of two independent experiments performed at $15^{\circ} \mathrm{C}$. Each time we scored at least 50 worms labeled with an antisense reagent. MT14119 contains a deletion of 1261 bases on chromosome II which removes mir-35 - mir-41 (ref. [8]). mir-42 with Rhdextran-(as-2'OMemir-42) 1 dramatically increased embryonic or L1 lethality: at the injection concentration of $3 \mu \mathrm{M}, 77 \%$ of labelled worms were embryonic or L1 lethal. In contrast, inhibition of mir-42 had little effect in N2 strain (Figure 9).

Among four miRNAs tested (lin-4, lsy-6, let-7 and mir42), the dose of antisense reagents required for the effective inhibition of individual miRNA varied from as low as three micromolar ( $l s y-6$ and mir-42) to as high as tens of micromolar (let-7). Two factors may account for the difference in the apparent potency of these antisense reagents. First, the cellular expression level of different miRNAs varies over a wide range [23], so a higher concentration of antisense reagents is needed to block more abundant miRNAs. Second, to inhibit a miRNA that is expressed late in larval development, it would require a higher concentration of reagent in the zygote to compensate for the dilution effect of cell division and larval growth. let-7, for example, is not expressed until the third larval stage and functions in the fourth stage [19]. By that time, the antisense reagent would be further diluted as animals grow and expand in size. In principle, a higher concentration of antisense reagents can be used to overcome the dilution effect. However, it should be cautioned that, as we raised the dose, we also noticed that an increasing number of embryos failed to hatch at concentrations above $50 \mu \mathrm{M}$. The extent to which these antisense reagents affect embryo development seemed to vary. Among the reagents tested, antisense reagents (antimirs) against lin-4 and mir-237 were best tolerated, with nearly $100 \%$ of the embryos hatched normally at $100 \mu \mathrm{M}$. By comparison, the antimir against let-7 was least tolerated, with close to $40 \%$ of embryos hatched at $100 \mu \mathrm{M}$ (Figure 10). We have not yet explored the causes for this differential effect, although we anticipate that the future development of other bioconjugates employing different classes of oligonucleotides, for example locked nucleic acids or 2'-O-methoxyethyl oligoribonucleotides, may offer an improvement on the potency with concomitant reduction of the perturbation on embryo development.

In order to test whether these antisense reagents can be used combinatorially to inhibit more than one miRNAs at a time, we coinjected Rhdextran-(as-2'OMelsy-6) ${ }_{1}$ and ${ }^{R h}$ dextran-(as-2'OMe $l i n-4)_{1}$ into gonads of OH3192 strain (gcy5 prom::gfp). Together, these two reagents caused Egl in all labelled worms and they induced ectopic $g c y$-5 prom::gfp expression in ASEL (Figure 11). In contrast, Rhdextran-(as2 'OMe $l i n-4)_{1}$ alone did not alter the expression pattern of $g c y-5$ prom::gfp, and $\left.{ }^{\mathrm{Rh}} \mathrm{dextran-({ } ^ { \text { as- } } 2 \mathrm { OMe }} l s y-6\right)_{1}$ by itself failed to cause Egl (Figure 11). The results again confirmed the specificity of these dextran-conjugated antisense reagents and suggested that multiple antisense reagents can be used in combination to block more than one miRNAs in 


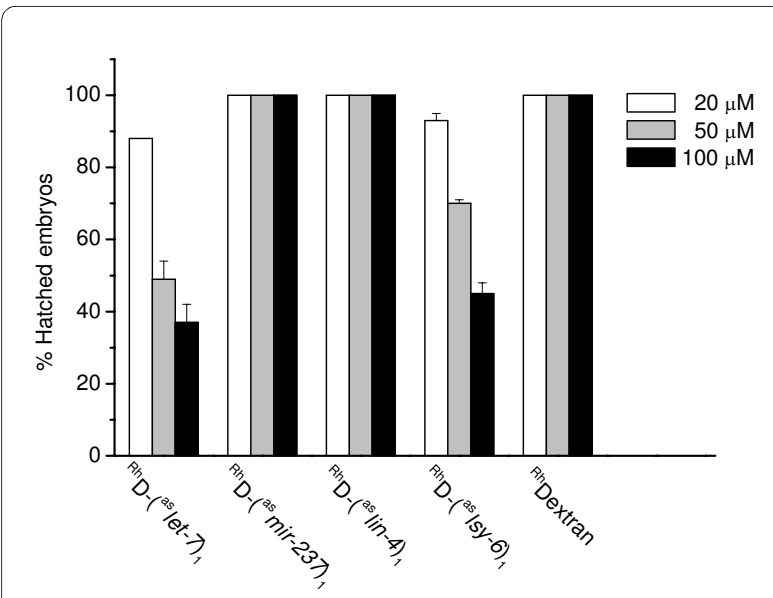

Figure 10 Variation of percentage of hatched embryos labeled with different doses of antisense reagents. Rhodamine-dextran ( $\mathrm{Rh}_{-}$ Dextran) or its conjugates of antisense 2'-O-methyl oligoribonucleotides against let-7, lin-4, 1sy-6, mir-237 were injected into gonads of N2 worms at three different concentrations. Each time we collected 50 or more labelled embryos and counted hatched larvae the next day. Error bars are standard errors of two independent injections. Examination of embryos that failed to hatch revealed that most of them were arrested around 50-cell stage. Embryos, once hatched, developed into adults that appeared to be grossly normal.

C. elegans. As numerous deletion strains of single miRNA genes appear to be grossly normal, it has been suggested that redundancy might mask their functions [8]. Mixing these antisense reagents would allow the study of the combinatorial effects of multiple miRNAs on gene expression and should facilitate screening genetic interactions using mutants or reporter strains.

\section{Conclusions}

We have developed a new class of antisense reagents that potently and selectively inhibit miRNAs in C. elegans. This offers an experimental approach complementary to mutational strategy for the study of the functions of miRNA in vivo.

\section{Methods}

\section{General methods}

2'-O-methyl oligoribonucleotides were either purchased from the Integrated DNA Technologies (IDT, Iowa, USA) or synthesized in-house by the standard solid phase phosphoramidite chemistry using an ABI 394 DNA/RNA Synthesizer (Applied Biosystems, California, USA). Sequences of 2'-O-methyl oligoribonucleotides used in this study are:

s-2'OMelin-4 (sense): 5' - UCCCUGAGACCUCAAGUGUGA - 3'

as-2'OMelin-4 (antisense): 5' - UCACACUUGAGGUCUCAGGGA - 3'

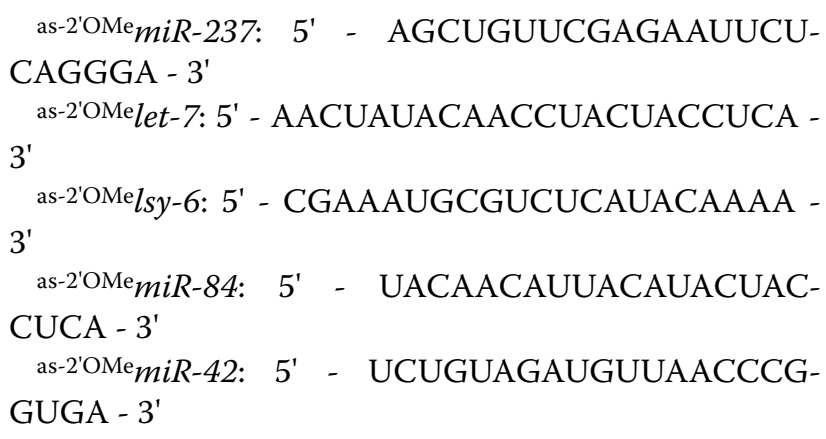

For bioconjugation, an $n$-hexyl linker containing a disulfide bond (Thio-Modifier C6 S-S, Glen Research, Virginia, USA) was attached to the 5'-end of 2'-O-methyl oligoribonucleotides. A,C,G,U-2'-OMe-RNA CE phosphoramidite monomers and A,C,G,U-2'-OMe RNA synthesis supports were from AZCO Biotech (California, USA). MAL-dPEG ${ }_{4}{ }^{\mathrm{m}}$-NHS ester was from Quanta BioDesign Ltd (Ohio, USA). Dextran amine (40 KD) was purchased from Molecular Probes (Oregon, USA). Other reagents and solvents were from Aldrich (Missouri, USA). The UV and visible absorption spectra were recorded on a Shimadzu 2401 PC spectrometer.

\section{Conjugating 2'-O-methyl oligonucleotides with dextran}

To prepare Rhdextran-(as-2'OMelin-4) ${ }_{1}$, for example, dextran amine (40 KD, 8 amines/dextran, $10 \mathrm{mg}$ ) was first reacted with Rhodamine B isothiocyanate (RBITC, 0.4 $\mathrm{mg}, 0.75 \mu \mathrm{mol}$ ) in $0.1 \mathrm{~mL}$ anhydrous DMSO at $37^{\circ} \mathrm{C}$ for 8 h. MAL-dPEG ${ }_{4}-\mathrm{NHS}$ ester $(3 \mathrm{mg}, 5.84 \mu \mathrm{mol})$ was then added and the reaction was continued at room temperature overnight. The reaction mixture was dialyzed against water through a regenerated cellulose membrane (Float A Lyzer, molecular wight cut off $[\mathrm{MWCO}]=3500$, Spectrum Laboratories, Inc. California, USA) to remove excess reagents. After freeze drying, the solid product was dissolved in water $(0.25 \mathrm{~mL})$ to make a $1 \mathrm{mM}$ rhodamine-dextran stock solution.

To conjugate 2'-O-methyl oligoribonucleotides containing a 5'-disulfide (5' S-S) group with rhodamine-dextran, we first reduced the 5'-disulfide group to a free thiol using tris(2-carboxyethyl) phosphine (TCEP), a water soluble reducing reagent. as-2'OMelin-4 (5' S-S, $30 \mathrm{nmol}$ ) was dissolved in $100 \mu \mathrm{l}$ of deaerated sodium phosphate buffer $(100 \mathrm{mM}, \mathrm{pH}=7.0)$. An excess amount of TCEP was added to the solution under the protection of Argon. One hour later, cold ethanol $(0.5 \mathrm{~mL})$ was added to precipitate the oligonucleotide. The supernatant was removed after centrifugation (14000 rpm for $10 \mathrm{~min}$ ) and the precipitated oligonucleotide was redissolved in a sodium phosphate buffer $(100 \mathrm{mM}, \quad \mathrm{pH}$ 7.0, $70 \mu \mathrm{L})$. The oligonucleotide solution was then mixed with the rhodamine-dextran stock solution $(30 \mu \mathrm{L})$ prepared above. 
a

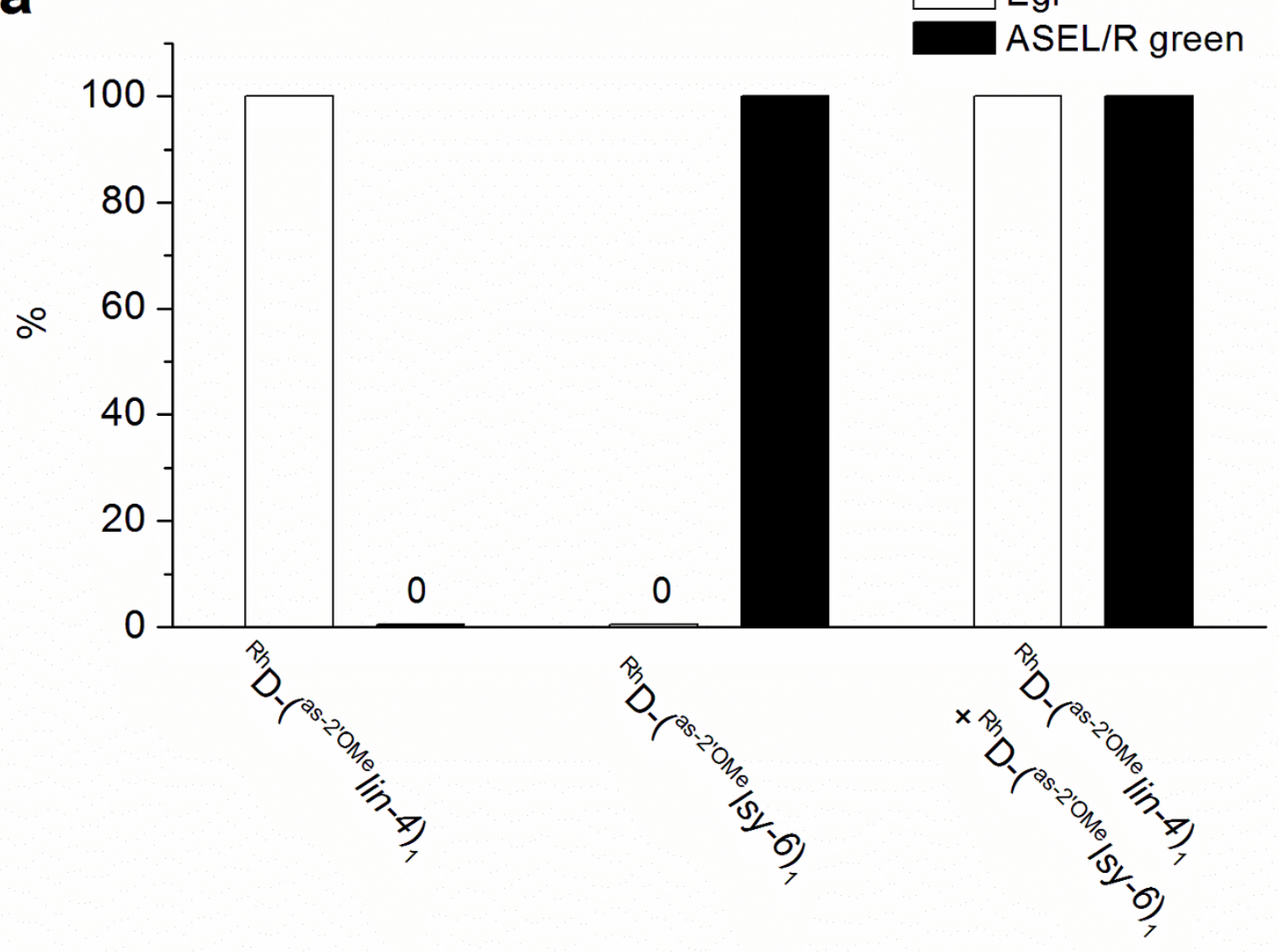

b

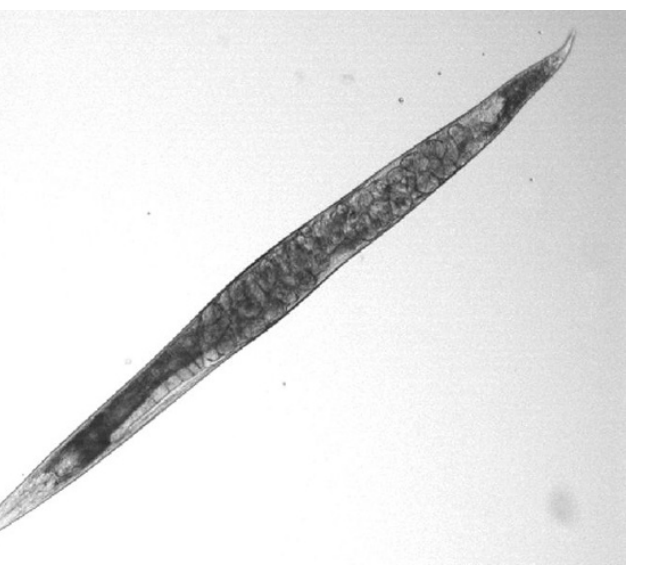

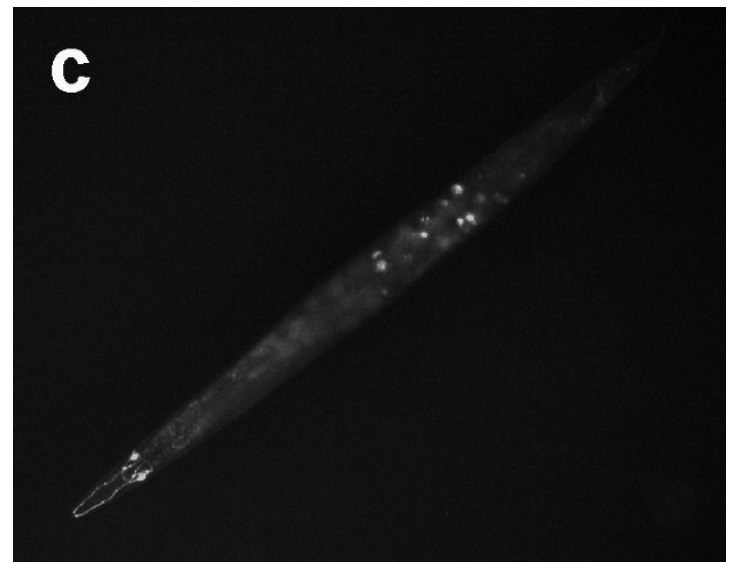

Figure 11 Concurrent inhibition of lin-4 and Isy- 6 in Caenorhabditis elegans with two antisense reagents. (a) Rh dextran-(as-2'OMe/in-4) ${ }_{1}(50 \mu M M)$ or Rh dextran-(as-2'OMe $/ s y-6)_{1}(20 \mu \mathrm{M})$, or a mixture of both reagents, were injected into gonads of OH3192 strain (gcy-5prom::gfp). The egg laying defective (Egl) and ectopic expression of gcy-5prom::.gfp in ASEL (left ASE) were scored ( $n=50$ worms. Error bars are standard errors of two independent injection experiments). (b, c) Bright field [(b) showing Egl] and fluorescence [(c) showing ectopic expression of gcy-5prom::gfp in ASEL] images of a worm (OH3192) labelled with both Rhdextran-(as-2'OMe/in-4), and Rhdextran-(as-2'OMe/sy-6) ${ }_{1}$. 
The mixture was stirred under argon overnight. Excess mercaptoethanol was added to cap the remaining unreacted maleimide group. The reaction mixture was dialyzed against water using a cellulose membrane (MWCO $=10,000)$ and lyophilized to yield the final product. The dried product was re-dissolved in water to prepare a stock solution. The concentration of stock solution was typically in the range of $1 \mathrm{mM}$ which was determined by measuring the UV absorption of 2'-O-methyl oligoribonucleotide at $260 \mathrm{~nm}$. The UV absorption was converted to the oligonucleotide concentration using the OligoAnalyzer program accessible online http://www.idtdna.com/ analyzer/Applications/OligoAnalyzer/. Rhodamine absorption at $260 \mathrm{~nm}$ was corrected according to its peak absorption at $559 \mathrm{~nm}$. The conjugation yields were typically around $50 \%$. The average stoichiometry of conjugation was calculated from the total amount of oligoribonucleotide in the final product divided by the amount of dextran added to the reaction.

RhDextran-(as-2'OMe $l i n-4)_{4}$, dextran-(as-2'OMe $\left.l i n-4\right)_{8}$ and other dextran conjugates containing different sequences of 2'-O-methyl oligonucleotides were synthesized similarly. When preparing RhDextran-(as-2'OMe $l i n-4)_{4}$, four equivalents of 2'-O-methyl oligonucleotides containing a 5 -disulfide group were used to react with the dextranlinked maleimide group. No Rhodamine B isothiocyanate was used when synthesizing dextran-(as-2'OMe $/$ in-4) $)_{8}$.

Conjugation products purified by dialysis still contained a small amount of unconjugated oligonucleotides as analyzed by the polyacrylamide gel electrophoresis (PAGE), and were used for most of the experiments except for the ones shown in Figure 8 and Figure 10. To completely remove unconjugated oligonucleotides from dextran-conjugates, the reaction mixture was first concentrated under vacuum to a small volume $(\leq 30 \mu \mathrm{L})$ and then mixed with $0.27 \mathrm{~mL}$ formamide (>99\%, Ambion, Texas, USA). The mixture was boiled briefly and loaded into $5 \%$ preparative denaturing polyacrylamide gel. After running the gel at $400 \mathrm{~V}$ for $10 \mathrm{~min}$, we confirmed the separation of free 2'-O-methyl oligoribonucleotides from dextran conjugates by viewing the gel over a fluorophorecoated thin-layer chromatography plate (Silica Gel 60, F254, Merck, Germany) under the UV illumination (265 $\mathrm{nm})$. Dextran-conjugated products that remained near the origin of the gel showing red fluorescence were cut out and transferred into a dialysis membrane $(\mathrm{MWCO}=$ 1000) containing $2 \mathrm{~mL}$ of $0.5 \times$ TBE buffer (Bio-Rad, CA, USA). The dialysis membrane was sealed and the product in the gel was recovered by electrophoresis $(300 \mathrm{~V}$ for 20 min). The TBE buffer in the dialysis membrane containing dextran conjugates was transferred to another cellulose dialysis membrane $(\mathrm{MWCO}=10000)$ and dialyzed against water 3 times over $16 \mathrm{hrs}$ to remove salts and urea. The final products were obtained as a powder after lyophilization.

\section{Worm injection and assay of miRNA inhibition in vivo}

Dextran conjugates of 2'-O-methyl oligoribonucleotides were injected into both gonads of young adult worms of either wild-type (N2) or transgenics expressing GFP in an ASE neuron (OH3191 or OH 3192). Rhodamine dextran (40 KD, $8 \mathrm{mg} / \mathrm{mL}$ final concentration) was included in the injection solution if the injected reagents contained no fluorescent label, for example, dextran-(as-2'OMelin-4) $)_{8}$ or unconjugated 2'-O-methyl oligoribonucleotides. For each experiment, we routinely injected a sample into $\sim$ eight worms. About $16 \mathrm{~h}$ later, we collected rhodamine labelled embryos $(n=50)$ laid by injected worms under a fluorescence dissection scope (SteREO Discovery.V12, Carl Zeiss, Göttingen, Germany), and scored their phenotypes when they reached appropriate larval or adult stages. Staging of animal development was based on gonad size and morphology.

We also attempted delivering antisense reagents using a standard soaking method for RNAi ([24]. However, this method turned out to be ineffective. After incubating L1 larvae with Rhdextran-(as-2'OMelin-4) ${ }_{1}(300 \mu \mathrm{M})$ or Rhdextran-(as-2'OMelet-7) $)_{1}(300 \mu \mathrm{M})$ for $48 \mathrm{~h}$ in the soaking solution (M9 solution $\left(0.25 \times\right.$, without $\left.\mathrm{Mg}^{2+}\right)$ with $3 \mathrm{mM}$ spermidine and $0.05 \%$ gelatin), we recovered L1 larvae on NGM plates. All the worms developed normally without showing any observable phenotype expected from lin-4 or let-7 knockdown.

\section{Abbreviations}

ASEL: left ASE; ASER: right ASE; Egl: egg laying defective; GFP: green fluorescence protein; MWCO: molecular weight cut off; TCEP: tris(2-carboxyethyl) phosphine; UV: ultraviolet; 3'UTR: 3'-untranslated region.

\section{Competing interests}

The authors declare that they have no competing interests.

\section{Authors' contributions}

GHZ and WHL designed the conjugates and syntheses. VA and WHL designed biological assays. GHZ performed all the experiments. WHL conceived the project. WHL, VA and GHZ analyzed the data and wrote the manuscript.

\section{Acknowledgements}

We thank the Caenorhabditis Genetics Center for providing worm strains and Dr X Wang for providing the microinjection apparatus. We are also grateful to Dr J Liu for help with the microinjection. This project is supported by the grants from the Welch Foundation (I-1510) and the National Institute of Health.

\section{Author Details}

1Departments of Cell Biology and of Biochemistry, University of Texas Southwestern Medical Center, 5323 Harry Hines Blvd, Dallas, TX 75390-9039, USA and 2Program in Molecular Medicine, University of Massachusetts Medical School, Worcester, MA 01605, USA

Received: 18 July 2009 Accepted: 1 April 2010

Published: 1 April 2010 


\section{References}

1. Ambros V: The functions of animal microRNAs. Nature 2004 431(7006):350-355

2. Hutvagner G, Simard MJ, Mello CC, Zamore PD: Sequence-specific inhibition of small RNA function. PLOS Bio/ 2004, 2(4):465-473.

3. Orom UA, Kauppinen S, Lund AH: LNA-modified oligonucleotides mediate specific inhibition of microRNA function. Gene 2006:137-141.

4. Esau C, Kang X, Peralta E, Hanson E, Marcusson EG, Ravichandran LV, Sun Y, Koo S, Perera RJ, Jain R, Dean NM, Freier SM, Bennett CF, Lollo B, Griffey R: MicroRNA-143 regulates adipocyte differentiation. J Biol Chem 2004, 279(50):52361-52365

5. Flynt AS, Li N, Thatcher EJ, Solnica-Krezel L, Patton JG: Zebrafish miR-214 modulates Hedgehog signaling to specify muscle cell fate. Nat Genet 2007, 39(2):259-263.

6. Boutla A, Delidakis C, Tabler M: Developmental defects by antisensemediated inactivation of micro-RNAs 2 and 13 in Drosophila and the identification of putative target genes. Nucleic Acids Res 2003, 31(17):4973-4980

7. Krutzfeldt J, Rajewsky N, Braich R, Rajeev KG, Tuschl T, Manoharan M, Stoffel M: Silencing of microRNAs in vivo with 'antagomirs'. Nature 2005, 438(7068):685-689

8. Miska EA, Alvarez-Saavedra E, Abbott AL, Lau NC, Hellman AB, McGonagle SM, Bartel DP, Ambros VR, Horvitz HR: Most Caenorhabditis elegans microRNAs are individually not essential for development or viability. PLoS genetics 2007, 3(12):2395-2403.

9. Ying SY, Lin SL: Intronic microRNAs. Biochem Biophys Res Commun 2005, 326(3):515-520

10. Mello CC, Kramer JM, Stinchcomb D, Ambros V: Efficient gene transfer in C.elegans: extrachromosomal maintenance and integration of transforming sequences. Embo J 1991, 10(12):3959-3970.

11. Guo YM, Chen S, Shetty P, Zheng G, Lin R, Li WH: Imaging dynamic cellcell junctional coupling in vivo using Trojan-LAMP. Nat Methods 2008, 5(9):835-841.

12. Lee RC, Feinbaum RL, Ambros V: The C. elegans heterochronic gene lin-4 encodes small RNAs with antisense complementarity to lin-14. Cell 1993, 75(5):843-854.

13. Wightman B, Ha I, Ruvkun G: Posttranscriptional regulation of the heterochronic gene lin-14 by lin-4 mediates temporal pattern formation in C. elegans. Cell 1993, 75(5):855-862.

14. Liu Z, Kirch S, Ambros V: The Caenorhabditis elegans heterochronic gene pathway controls stage-specific transcription of collagen genes. Development 1995, 121(8):2471-2478.

15. Abrahante JE, Miller EA, Rougvie AE: Identification of heterochronic mutants in Caenorhabditis elegans. Temporal misexpression of collagen::green fluorescent protein fusion gene. Genetics 1998, 149(3):1335-1351.

16. Rougvie AE: Control of developmental timing in animals. Nature Rev Genet 2001, 2(9):690-701.

17. Ambros V, Horvitz HR: The lin-14 locus of Caenorhabditis elegans controls the time of expression of specific postembryonic developmental events. Genes Dev 1987, 1(4):398-414.

18. Hobert O: Architecture of a microRNA-controlled gene regulatory network that diversifies neuronal cell fates. Cold Spring Harb Symp Quant Biol 2006, 71:181-188.

19. Reinhart BJ, Slack FJ, Basson M, Pasquinelli AE, Bettinger JC, Rougvie AE, Horvitz HR, Ruvkun G: The 21-nucleotide let-7 RNA regulates developmental timing in Caenorhabditis elegans. Nature 2000, 403(6772):901-906.

20. Abbott AL, Alvarez-Saavedra E, Miska EA, Lau NC, Bartel DP, Horvitz HR, Ambros $\mathrm{V}$ : The let-7 MicroRNA family members mir-48, mir-84, and mir241 function together to regulate developmental timing in Caenorhabditis elegans. Dev Cell 2005, 9(3):403-414.

21. Lau NC, Lim LP, Weinstein EG, Bartel DP: An abundant class of tiny RNAs with probable regulatory roles in Caenorhabditis elegans. Science 2001, 294(5543):858-862.

22. Alvarez-Saavedra EA, Miska EA, Abbott AL, Lau NC, Bartel DP, Ambros V, Horvitz HR: The mir-35 family of MicroRNAs acts redundantly in embryonic development in C. elegans. International Worm Meeting: abstract 2692005.

23. Lim LP, Lau NC, Weinstein EG, Abdelhakim A, Yekta S, Rhoades MW, Burge CB, Bartel DP: The microRNAs of Caenorhabditis elegans. Genes Dev 2003, 17(8):991-1008.
24. Timmons L: Delivery methods for RNA interference in C. elegans. Methods Mol Biol 2006, 351:119-125.

doi: 10.1186/1758-907X-1-9

Cite this article as: Zheng et al., Inhibiting miRNA in Caenorhabditis elegans using a potent and selective antisense reagent Silence 2010, 1:9

\section{Submit your next manuscript to BioMed Central and take full advantage of:}

- Convenient online submission

- Thorough peer review

- No space constraints or color figure charges

- Immediate publication on acceptance

- Inclusion in PubMed, CAS, Scopus and Google Scholar

- Research which is freely available for redistribution 